\title{
Unitarity Triangle Analysis and D meson mixing in the Standard Model and Beyond
}

\author{
Marcella Bona* \\ Queen Mary University of London \\ E-mail: m.bona@ qmul . ac.uk \\ Luca Silvestrini \\ INFN Sezione of Roma \\ E-mail: Luca.Silvestrini@romal.infn.it \\ on behalf of the $\mathbf{U} \mathbf{T}$ fit Collaboration ${ }^{\dagger}$
}

Flavour physics represents a unique test bench for the Standard Model (SM). New analyses performed at the LHC experiments are now providing unprecedented insights into CKM metrology and new evidences for rare decays. The CKM picture can provide very precise SM predictions through global analyses. We present here the results of the latest global SM analysis performed by the UIfit collaboration including all the most updated inputs from experiments, lattice QCD and phenomenological calculations. In addition, we update the analysis of $D$ meson mixing: we derive constraints on the parameters $M_{12}, \Gamma_{12}$ and $\Phi_{12}$ that describe $D$ meson mixing using all available data, allowing for CP violation. Finally, the Unitarity Triangle (UT) analysis can be used to constrain the parameter space in possible new physics (NP) scenarios. All of the available experimental and theoretical information on $\Delta F=2$ processes is reinterpreted including a modelindependent NP parametrisation. We determine the allowed NP contributions in the kaon, $D, B_{d}$, and $B_{S}$ sectors and, in various NP scenarios, we translate them into bounds for the NP scale as a function of NP couplings.

The European Physical Society Conference on High Energy Physics

5-12 July, 2017

Venice

\footnotetext{
${ }^{*}$ Speaker.

${ }^{\dagger}$ The UIfit Collaboration: Cristiano Alpigiani (University of Washington), Adrian Bevan (Queen Mary University of London), Marcella Bona (Queen Mary University of London), Marco Ciuchini (INFN Sezione di Roma Tre), Denis Derkach (Yandex/Higher School of Economics), Enrico Franco (INFN Sezione di Roma), Vittorio Lubicz (University of Roma Tre), Guido Martinelli (University of Roma La Sapienza), Fabrizio Parodi (University of Genova and INFN), Maurizio Pierini (CERN), Luca Silvestrini (INFN Sezione di Roma), Viola Sordini (IPNL-IN2P3 Lyon), Achille Stocchi (LAL-IN2P3 Orsay), Cecilia Tarantino (University of Roma Tre), Vincenzo Vagnoni (INFN Sezione di Bologna).
} 

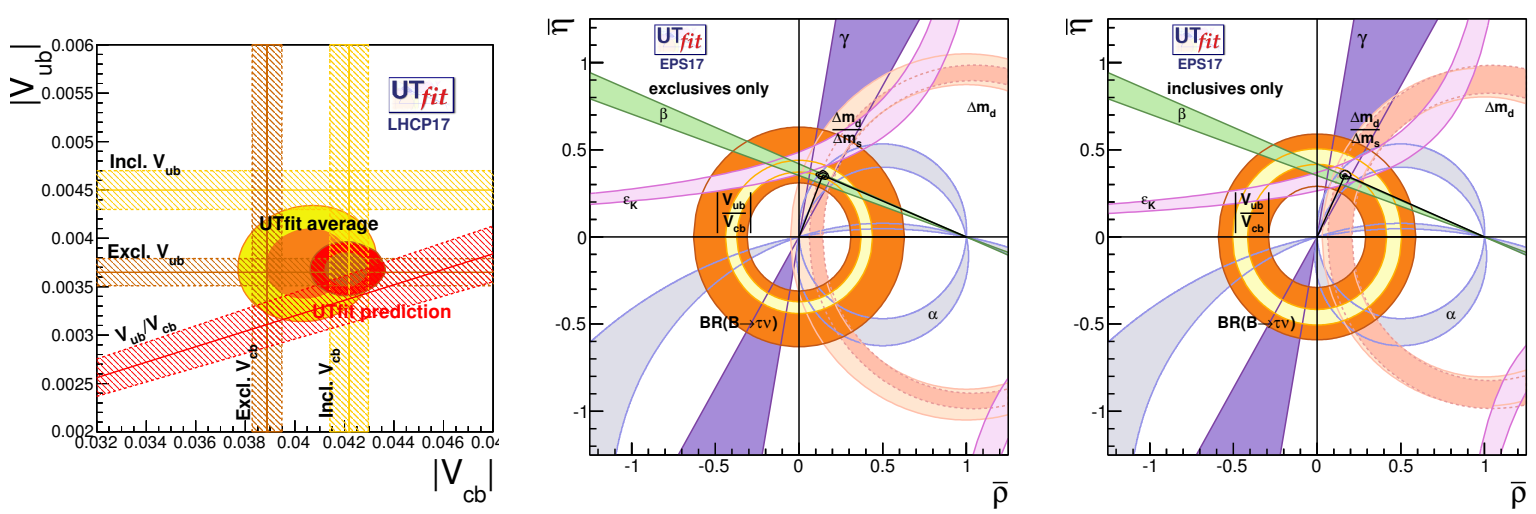

Figure 1: Left: $\left|V_{c b}\right|$ vs $\left|V_{u b}\right|$ plane showing the values reported in Table 1. We include in the average the LHCb ratio measurement [7] that is shown as a diagonal band. Centre-Right: $\bar{\rho}-\bar{\eta}$ plane with the SM global fit results using only exclusive inputs for both $V_{u b}$ and $V_{c b}$ (centre) and using only inclusive inputs (right).

\section{Introduction}

Flavour physics represents a powerful tool to test the Standard Model (SM), to quantify the coherence of its picture and to explore possible departures from it. From the flavour global fit we can extract the most accurate determination of the parameters of the CKM matrix [1], as well as the best SM predictions of flavour observables. The Unitarity Triangle (UT) analysis here presented is performed by the $\mathbf{U} \mathbf{T}$ fit Collaboration following the method described in Refs. [2]. We updated the analysis with the latest determinations of the theoretical inputs and the latest measurements of the experimental observables. The basic constraints used in the global fit and contributing to the sensitivity of the CKM matrix elements are: $\left|V_{u b} / V_{c b}\right|$ from semileptonic $B$ decays, $\Delta m_{d}$ and $\Delta m_{s}$ from $B_{d, s}^{0}$ oscillations, $\varepsilon_{K}$ from neutral $K$ mixing, $\alpha \mathrm{UT}$ angle from charmless hadronic $B$ decays, $\gamma$ UT angle from charm hadronic $B$ decays, and the sine of $2 \beta$ UT angle from $B^{0} \rightarrow J / \psi K^{0}$ decays.

The values of most experimental inputs are taken from the Heavy Flavour Averaging Group (HFLAV) [3], however when most updated individual results are available the UT $f$ it collaboration performs its own averages. Below a specific update is discussed for the $\left|V_{u b} / V_{c b}\right|$ experimental input. On the theoretical side, the non-perturbative QCD parameters are taken from the most recent lattice QCD determinations: as a general prescription, we average the $N_{f}=2+$ $1+1$ and $N_{f}=2+1$ FLAG numbers [4], using eq. (28) in Ref. [5] and including the results in Ref. [6]. The continuously updated set of numerical values used as inputs can be found at URL http://www.utfit.org/.

\section{Updated inputs and results of the global fit in the SM}

For the inputs coming from the semileptonic $B$ decays, we use the values shown in the left plot

Table 1: $V_{c b}$ and $V_{u b}$ experimental inputs are shown as values. The individual $V_{c b}$ and $V_{u b}$ exclusive and inclusive numbers are taken from the most updated HFLAV averages [3].

\begin{tabular}{ccc|c|c}
{$\left[10^{-3}\right]$} & excl. & incl. & $\left|\mathbf{V}_{\mathbf{u b}} /\right| \mathbf{V}_{\mathbf{c b}} \mid$ & 2D average \\
$\left|\mathbf{V}_{\mathbf{c b}}\right|$ & $38.88 \pm 0.60$ & $42.19 \pm 0.78$ & $(8.0 \pm 0.6) 10^{-2}$ & $40.5 \pm 1.1$ \\
$\left|\mathbf{V}_{\mathbf{u b}}\right|$ & $3.65 \pm 0.14$ & $4.50 \pm 0.20$ & & $3.74 \pm 0.23$
\end{tabular}



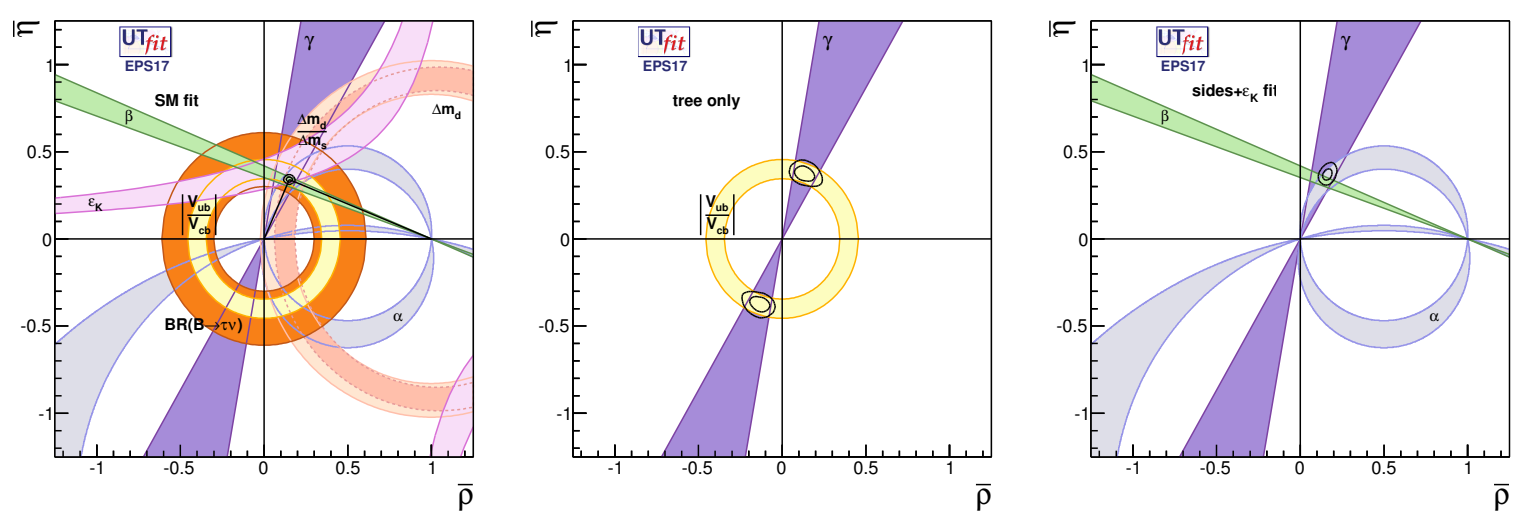

Figure 2: $\bar{\rho}-\bar{\eta}$ planes showing the result of the full SM fit (left), the result of the tree-only fit (centre) and the result of the SM fit using the UT sides and the kaon mixing compared with the areas of the UT angle constraints (right). The black contours display the $68 \%$ and $95 \%$ probability regions selected by the given global fit. The $95 \%$ probability regions selected are also shown for each constraint considered.

Table 2: Results of the fit to $D$ mixing data.

\begin{tabular}{|cc|cc|}
\hline parameter & result @ 68\% prob. & parameter & result @ 68\% prob. \\
\hline$\left|M_{12}\right|\left[\mathrm{ps}^{-1}\right]$ & $(4.3 \pm 1.8) \cdot 10^{-3}$ & $x$ & $(3.5 \pm 1.5) \cdot 10^{-3}$ \\
$\left|\Gamma_{12}\right|\left[\mathrm{ps}^{-1}\right]$ & $(14.2 \pm 1.4) \cdot 10^{-3}$ & $y$ & $(5.8 \pm 0.6) \cdot 10^{-3}$ \\
$\Phi_{M_{12}}\left[^{\circ}\right]$ & $(0.3 \pm 2.6)$ & $|q / p|-1$ & $0.002 \pm 0.018$ \\
$\phi\left[^{\circ}\right]$ & $-0.08 \pm 0.57$ \\
\hline
\end{tabular}

in Fig. 1 and listed in Table 1. The UTfit two-dimensional (2D) average shown is calculated with a 2D procedure inspired by the skeptical method of Ref. [8] with $\sigma=1$. It is evident that exclusive and inclusive results persist in showing discrepancies at the level of about $3.3 \sigma$ in the case of $V_{c b}$ and about $3.4 \sigma$ for $V_{u b}$. The effect of these deviations in the global fit results are shown in the right plots in Fig. 1. These inclusive-vs-exclusive discrepancies have been highlighted and discussed by the UIfit collaboration since 2006 [9].

Using the latest inputs and our Bayesian framework, we perform the global fit to extract the CKM matrix parameters $\bar{\rho}$ and $\bar{\eta}$ : we obtain $\bar{\rho}=0.151 \pm 0.014$ and $\bar{\eta}=0.342 \pm 0.013$. The left plot in Fig. 2 shows the result of the SM fit on the $\bar{\rho}-\bar{\eta}$ plane, while the central figure shows the "tree-only" fit when only tree-level measurements are included $\left(\left|V_{u b} / V_{c b}\right|\right.$ and $\gamma$ assumed NP-free). Fig. 2 right plot shows the comparison between the areas coming from the angle measurements and the fit result obtained using all the other constraints (the UT sides and the kaon mixing $\varepsilon_{K}$ ). The main tension still present in the global fit comes from the inclusive-vs-exclusive values of the semileptonic determinations: for example, the inclusive $\left|V_{u b}\right|$ value shows a $\sim 3.8 \sigma$ discrepancy with respect to the rest of the fit.

We update here also the fit to the $D$ mixing experimental data that are reported in Table 1 of the 2014 Ref. [10]: Table 2 here shows the results updated in the 2017 analysis, following the statistical method described in Ref. [2] improved with a Markov-chain Monte Carlo as implemented in the BAT library [11]. The input averages are taken from the Heavy Flavour Averaging Group (HFLAV) [3]. 

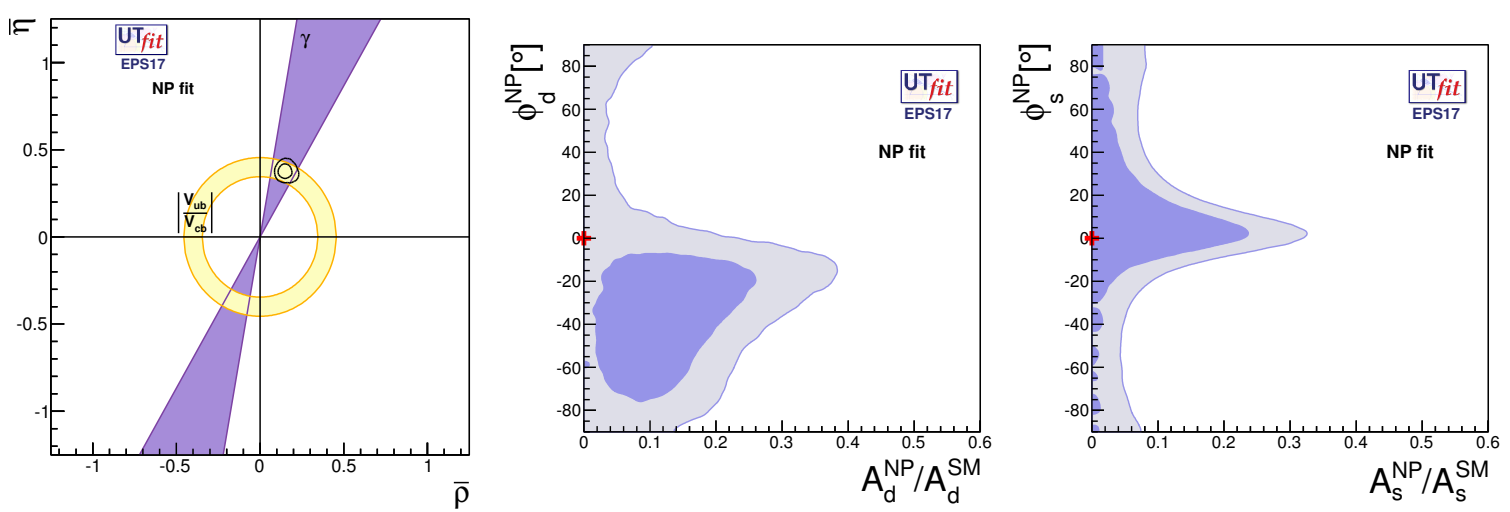

Figure 3: Left: $\bar{\rho}-\bar{\eta}$ plane showing the result of the NP fit. The black contours display the $68 \%$ and $95 \%$ probability regions selected by the NP global fit. The $95 \%$ probability regions selected are shown for those constraints not affected by NP in $\Delta F=2$ transitions, however all the constraints are used in the fit selecting the $\bar{\rho}-\bar{\eta}$ area. Centre-right: $A_{q}^{\mathrm{NP}} / A_{q}^{\mathrm{SM}}-\phi_{q}^{\mathrm{NP}} \mathrm{NP}$-parameter plane as selected from the NP fit (left) for the $B_{d}$ system and (right) for the $B_{s}$ system, where $68 \%$ (dark) and $95 \%$ (light) probability regions are shown. The red cross represents the SM expectation.

\section{Result of the global fit beyond the SM}

We now consider the UT analysis performed reinterpreting the experimental observables including possible model-independent NP contributions. The NP effects considered are those entering the neutral meson mixing ( $\Delta F=2$ transitions). They are parameterised in a general way as a NP amplitude $A_{q}^{\mathrm{NP}}$ and a NP phase $\phi_{q}^{\mathrm{SM}}$, where $q=d$ or $s$ and in the SM it is $A_{q}^{\mathrm{NP}}=0$ and $\phi_{q}^{\mathrm{NP}}=0$.

We perform the NP analysis and the result of the NP global fit selects a region in the $(\bar{\rho}, \bar{\eta})$ plane which is consistent with the result of the SM analysis. This is shown in the left plot in Fig. 3. The $\bar{\rho}$ and $\bar{\eta}$ values extracted from the NP global fit are $\bar{\rho}=0.154 \pm 0.029$ and $\bar{\eta}=0.377 \pm 0.029$. Simultaneously, the NP parameters are extracted and their allowed ranges are shown in the two right plots of Fig. 3. The current tension of the SM picture is reflected in the $B_{d}$ sector. In general a $30-40 \% \mathrm{NP}$ effect is allowed at $95 \%$ probability, given the current sensitivities.

If we consider the most general effective Hamiltonian for $\Delta F=2$ processes $\left(H_{\mathrm{eff}}^{\Delta F=2}\right)$, we can translate the current constraints into allowed ranges for the Wilson coefficients of $H_{\text {eff }}^{\Delta F=2}$. The full procedure and analysis details are given in [12]. These coefficients have the general form $C_{i}(\Lambda)=F_{i} L_{i} / \Lambda^{2}$, where $F_{i}$ is a function of the (complex) NP flavour couplings, $L_{i}$ is a loop factor that is present in models with no tree-level Flavour Changing Neutral Currents, and $\Lambda$ is the scale of NP, i.e. the typical mass of the new particles mediating $\Delta F=2$ transitions. For a generic strongly-interacting theory with arbitrary flavour structure, one expects $F_{i} \sim L_{i} \sim 1$ so that the allowed range from the fit for each of the $C_{i}(\Lambda)$ can be immediately translated into a lower bound on $\Lambda$. Specific assumptions on the flavour structure of NP, for example Next-to-Minimal [13] Flavour Violation (NMFV), correspond to particular choices of the $F_{i}$ functions. In the case of NMFV, we have $\left|F_{i}\right|=F_{\text {SM }}$ with an arbitrary phase [13]. To obtain the lower bound on $\Lambda$ for loopmediated contributions, one simply multiplies the bounds we quote in the following by $\alpha_{s} \sim 0.1$ or by $\alpha_{W} \sim 0.03$.

In the case of the general NP scenario, we have arbitrary NP flavour structures $\left(\left|F_{i}\right| \sim 1\right)$ with arbitrary phase and $L_{i}=1$ corresponding to strongly-interacting and/or tree-level NP. The 
overall strongest constraint on the NP scale comes from the kaon sector and it is translated into $\Lambda_{\text {gen }}>5.0 \cdot 10^{5} \mathrm{TeV}$. As we are considering arbitrary NP flavour structures, the constraints on the NP scale are very tight due to the absence of the CKM suppression.

In the NMFV case, the strongest bound is again obtained from the kaon sector and it translated into the weaker lower limit $\Lambda_{\mathrm{NMFV}}>114 \mathrm{TeV}$. In this latter case and in the current scenario, the $B_{s}$ system also provides quite stringent constraints.

In conclusion, a loop suppression is needed in all scenarios to obtain NP scales that can be reached at the LHC. For NMFV models, an $\alpha_{W}$ loop suppression might not be sufficient, since the resulting NP scale is still of the order of $11 \mathrm{TeV}$. The general model is out of reach even for $\alpha_{W}$ (or stronger) loop suppression. Finally, the reader should keep in mind the possibility of accidental cancellations among the contribution of different operators, that might weaken the bounds we obtained.

\section{References}

[1] N. Cabibbo, Unitary symmetry and leptonic decays, Phys. Rev. Lett. 10 (Jun, 1963) 531-533; M. Kobayashi and T. Maskawa, CP Violation in the Renormalizable Theory of Weak Interaction, Prog.Theor.Phys. 49 (1973) 652-657.

[2] M. Ciuchini et al., 2000 CKM triangle analysis: A Critical review with updated experimental inputs and theoretical parameters, JHEP 0107 (2001) 013, [hep-ph/ 0012308 ]; UTFIT collaboration, M. Bona et al., The 2004 UTfit collaboration report on the status of the unitarity triangle in the standard model, JHEP 0507 (2005) 028, [hep-ph/ 0501199 ].

[3] Y. Amhis et al., Averages of b-hadron, c-hadron, and $\tau$-lepton properties as of summer 2016, 1612.07233.

[4] S. Aoki et al., Review of lattice results concerning low-energy particle physics, 1607.00299.

[5] European Twisted Mass collaboration, N. Carrasco et al., Up, down, strange and charm quark masses with $N_{f}=2+1+1$ twisted mass lattice QCD, Nucl. Phys. B887 (2014) 19-68, [1403.4504].

[6] Fermilab Lattice, MILC collaboration, A. Bazavov et al., $B_{(s)}^{0}{ }^{-m i x i n g ~ m a t r i x ~ e l e m e n t s ~ f r o m ~}$ lattice QCD for the Standard Model and beyond, Phys. Rev. D93 (2016) 113016, [1602. 03560].

[7] LHCB collaboration, R. Aaij et al., Determination of the quark coupling strength $\left|V_{u b}\right|$ using baryonic decays, Nature Phys. 11 (2015) 743-747, [1504.01568].

[8] G. D’Agostini, Sceptical combination of experimental results: General considerations and application to epsilon-prime / epsilon, Submitted to: Phys. Rev. D (1999), [hep-ex/ 9910036$].$

[9] UTFIT collaboration, M. Bona et al., The Unitarity Triangle Fit in the Standard Model and Hadronic Parameters from Lattice QCD: A Reappraisal after the Measurements of $\Delta m_{s}$ and $B R\left(B \rightarrow \tau \nu_{\tau}\right)$, JHEP 0610 (2006) 081, [hep-ph / 0606167 ].

[10] UTFIT collaboration, A. J. Bevan et al., The UTfit collaboration average of D meson mixing data: Winter 2014, JHEP 03 (2014) 123, [1402 . 1664].

[11] A. Caldwella, D. Kollar and K. Kroninger, BAT - The Bayesian analysis toolkit, Computer Physics Communications 180 (2009) 11, 2197-2209.

[12] UTFIT collaboration, M. Bona et al., Model-independent constraints on $\Delta F=2$ operators and the scale of new physics, JHEP $\mathbf{0 8 0 3}$ (2008) 049, [0 707. 0636].

[13] K. Agashe et al., Next to minimal flavor violation, hep-ph/0509117. 